\title{
Rotation of Scanner's Mirror in MEMS Dimensions with the Use of Lorents Law
}

\author{
Said Farahat ${ }^{1} \&$ Aria Nouri Jangi ${ }^{1}$ \\ ${ }^{1}$ University of Sistan and Baluchestan (Faculty of Engineering), Iran \\ Correspondence: Aria Nouri Jangi, University of Sistan and Baluchestan (Faculty of Engineering), Iran. E-mail: \\ aria.nouri@live.com
}

Received: January 20, 2016

Accepted: February 20, 2016

Online Published: February 29, 2016

doi:10.5539/mas.v10n3p201

URL: http://dx.doi.org/10.5539/mas.v10n3p201

\begin{abstract}
In this project we study a kind of scanner in micro dimensions in which it includes three frames also the third frame is considered as fixed surface and the two others are including two separate coils that electric current enters it and when we expose this electric current in a magnetic field under a specific conditions then with the use of Lorents Force law we can cause the rotation of the frame and mirror's surface accordingly.

Discussed field in this project has a magnitude of between 0.2-4 Tesla in which this magnetic field has no destructive effect on human body also the electric current has a magnitude of between 0.001-0.14 Amper in which the maximum obtained rotation angle is equal to \pm 54.49724 that in every half cycle with the use of two frames it can be deviated $\mathbf{5 4 . 4 9 7 2 4}$ degree from the mirror. This plan with the lower number in coil with a design in smaller dimensions (micrometer) is simulated with a more different design and the result was acceptable so it has been much favored for its medical capabilities.

Frames are linked together with Su-8 polymer in which this polymer has been used because of its appropriate softness and bending.
\end{abstract}

Keywords: MEMS, micro mirror, lorents force, su-8, scanner, magnetic field

\section{Introduction}

Today's demand of market, industry and medical science in the field of MEMS caused that instruments and micro tools has been attracted much interest.

Mirrors in micro dimensions has got the most industrial and medical applications so it has caused to much discuss about the rotation of the micro mirrors, methods and specific applications.

In this article we discuss about the rotation of the mirror with the use of Lorents Force law and SU-8 polymer.

SU-8 is used as a negative brapoxy contextual photoresistor. This polymer is very viscous which can rotate or can extend over 1 to 300 micrometer or more. The reason we use SU-8 polymer in this plan is the specific features of this polymer which can cause rotation, bending and high flexibility.

This project consists of three frames in which the third frame is static and fixed and all the three frames are linked together with SU-8 polymer and from the inside of SU-8 polymer a narrow wire is used to deliver electric current to inside of the frames. According to Lorents law when we expose a wire which has electric current into the magnetic field then there would appear a force in direction like the Figure below and magnitude of this force is calculated as below equation.

$\mathrm{F}=\mathrm{LxlxB}$

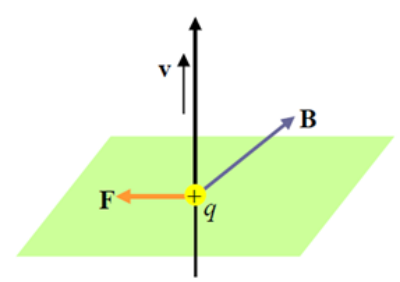

Figure 1. Lorents law for current-carrying wire which expose in a magnetic field 
According to this introduction we have three items to study the electric force in which the first item is $\mathrm{L}$ or the length of the wire but in case of this project because we can't change the length of the wire so we don't study wire length and its changes so the other two items are electric current and electric field that we study electric field and scale of the electric field changes on the scale of micro mirror rotation in here.

The range of electric field we study here is in between 0.2-5 Tesla in which has not showed destructive effect on human body or organisms and is used in MRI and CT instruments.

This project has been designed in Solidwork software and it has been reviewed in comsol software which is strong and multi physics software.

\section{Manufacturing Steps}

The third frame is made from Si3N4 in which the reason of selection is its high density and also its non-conductive feature so through this frame the electric current can enter the first and second frames.

For surface (plate) of the first and second frames we use three layers as per Figire 2

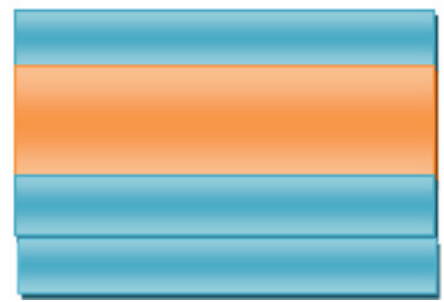

Figure 2. Plate of first and second frame which consist of a 20 micrometer width layer of silicon and two layers of 2 micrometer from silicon oxide. Length and width of first frame is shown in Figure. 2

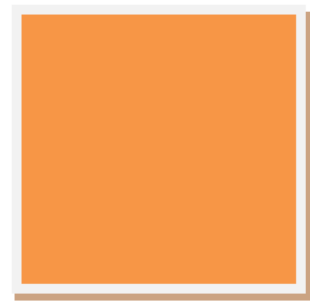

Figure 2. Length and width of first frame which is a square of 50 micrometer each side. Length and width of second frame is demonstrated in Figure 3.

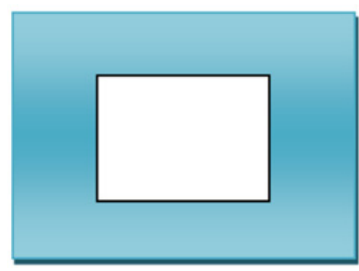

Figure 3. length and width of the second frame which is square and the inside square of 70 micrometer and outside square is 150 micrometer each side.

Project's external view is like Figure 4.

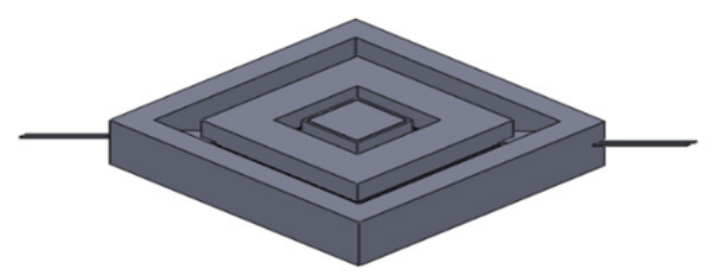

Figure 4. external view of project together with three frames and mirror and electric current input wires 
On the plate surface of first and second frames we cover 20 micrometer of SU-8 layer and then we use four numbers of coil on the surface to pass electric current wires made from gold and this is because of its conductivity and electric resistance and we can use other electric conductors which if we use other material then we can see the results will change.
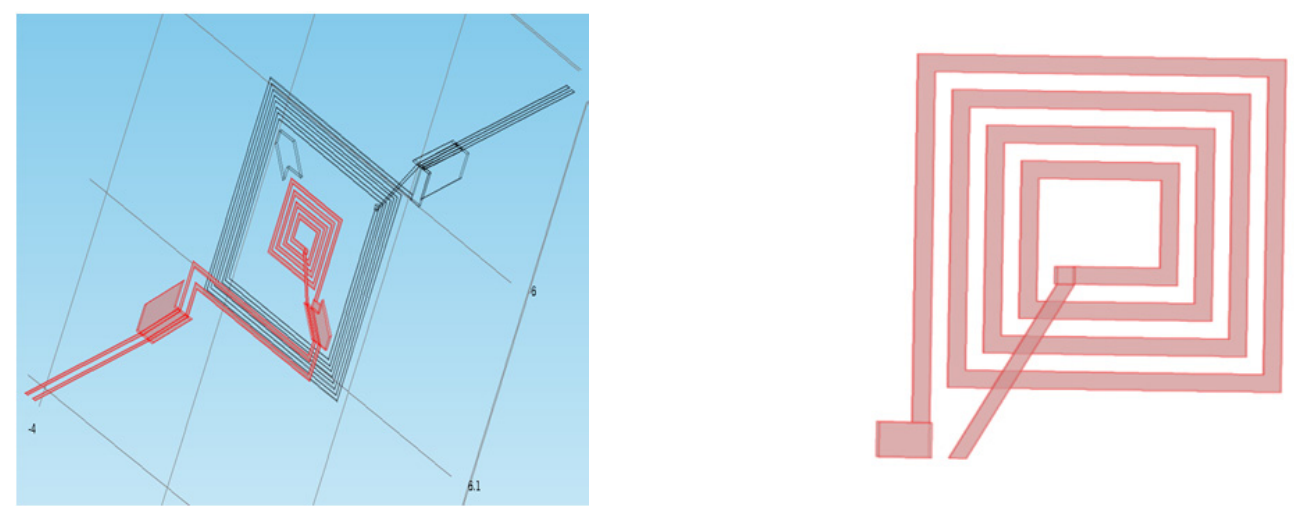

Figure 5. In right side the coil of the first frame and in left side the coil of the first and second frame as the main plan are showed

We cover the coil with SU-8 polymer. To make mirror,in the center of the first frame a gold surface is used in the center so that it can work as micro mirror.

The coil of the first frame and coil of the second frame are independent and separated from each other and transfer from SU-8 lobes and separately from first and second to third frame also exits from third frame to join resource and input of electric current.

Section form coefficient which is introduced as $\xi$ is obtained from below equation in which $1, \mathrm{~b}$ and a, are cross section, length and width of torsional rod of SU-8 and G is the shear elasticity module which for SU-8 polymer is equal to $2.056 \mathrm{~Pa}$ that is 3 percent of silicon article as bulk.

$$
\xi_{x}=\frac{1}{3}\left(1-\frac{192}{\pi^{5}} \frac{b_{x}}{a_{x}} \sum_{n=1}^{\infty} \frac{1}{(2 n-1)^{5}} \tanh \left(\frac{(2 n-1)}{2} \pi \frac{a_{x}}{b_{x}}\right)\right)
$$

Which generated torque is shown by $\mathrm{Kx}$ and is obtained from below equation

$$
K_{x}=\frac{\xi_{x} a_{x} b_{x}^{3}}{l_{x}} G
$$

And created angle by idx is obtained from below equation

$$
\theta_{x}=B \frac{1}{2 \sqrt{2}} \frac{R_{c x}^{2} i_{d x} n_{x}}{K_{x}}
$$

Which B is electric field and Rcx is mean length of the coil and Nx is the number of the coil.

By taking the span into consideration so the number of the meshes according to sensitivity of each section are divided into three types which we will face with 1149556 meshes in network's independency.

\section{Conclusion}

Passing of the force through coil of the first and second frame and existence of electric field cause to creation of the Lorents force and created Lorents force will be as below Figure's diagram. 


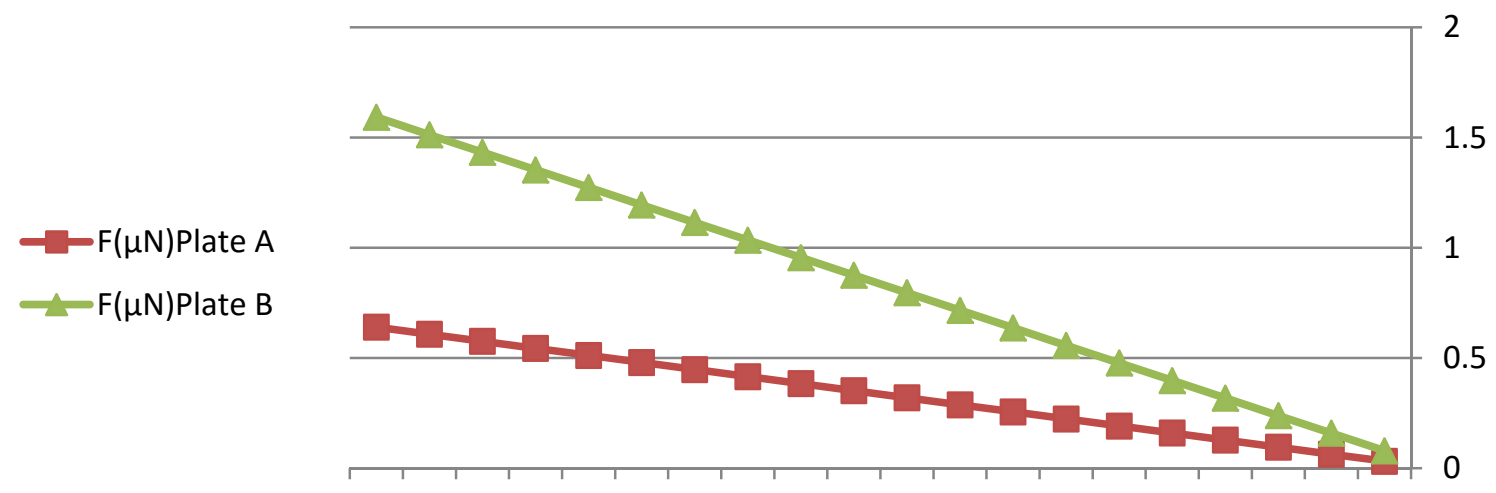

$4 \quad 3.83 .63 .43 .2 \quad 3 \quad 2.82 .62 .42 .2 \quad 2 \quad 1.81 .61 .41 .2 \quad 1 \quad 0.80 .60 .40 .2$

Figure 6. Lorents force on the first and second frame in field of 0.2-4 Tesla

By creating force in first and second frame it will cause rotation in first and second frame in which this rotation in frame will cause the rotation of the mirror. Rotation of the mirror in different fields and in input current of 0.01 Amper is like below.

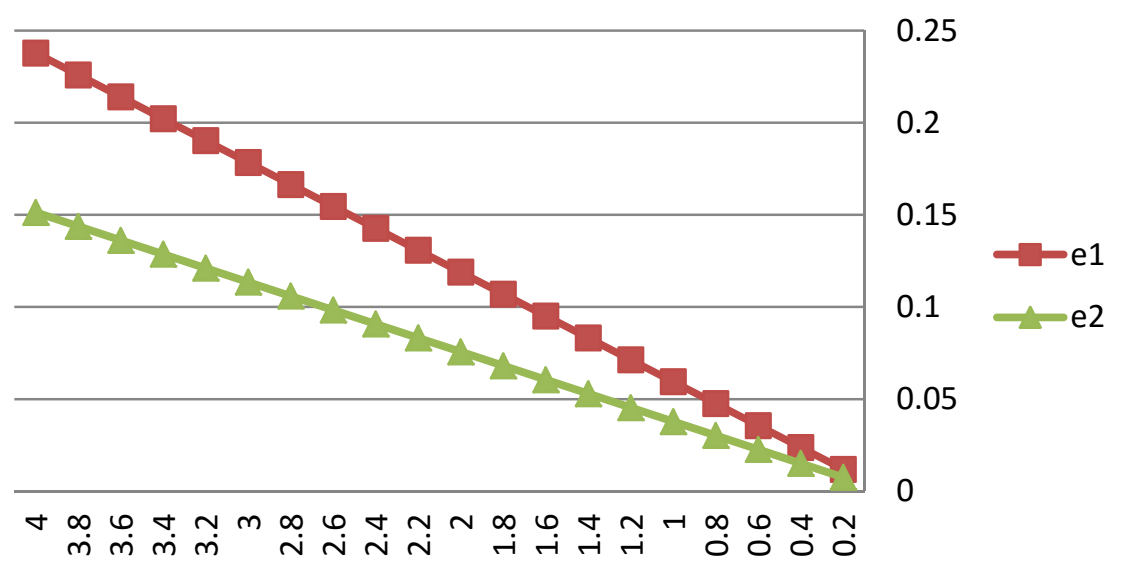

Figure 7. Changes of first and second frame's angle in 0.001 Amper current and field of 0.2-4 Tesla

And in fields of 2,3 and 4 Tesla and with a increase in current will be like below which the maximum of the angle in field of 4 Tesla and current of 0.14 Amper will happen and the mirror will rotate as \pm 54.49724 .

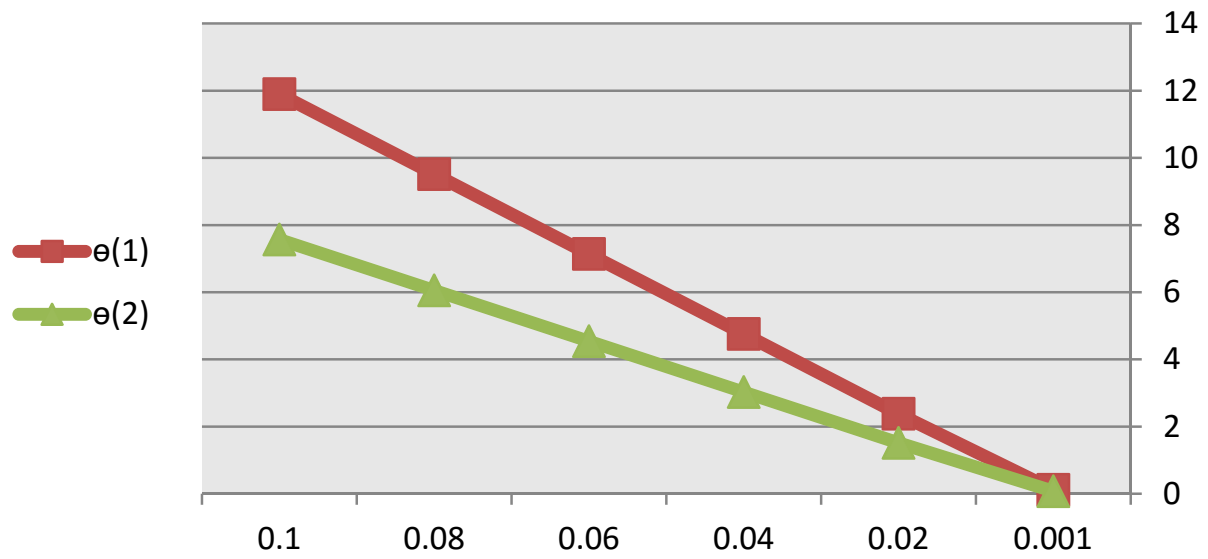

Figure 8. Vertical axis is angle and horizontal axis is electric current in the fixed field of 2 Tesla and diagram of first and second frames angle changes is different in electric currents 


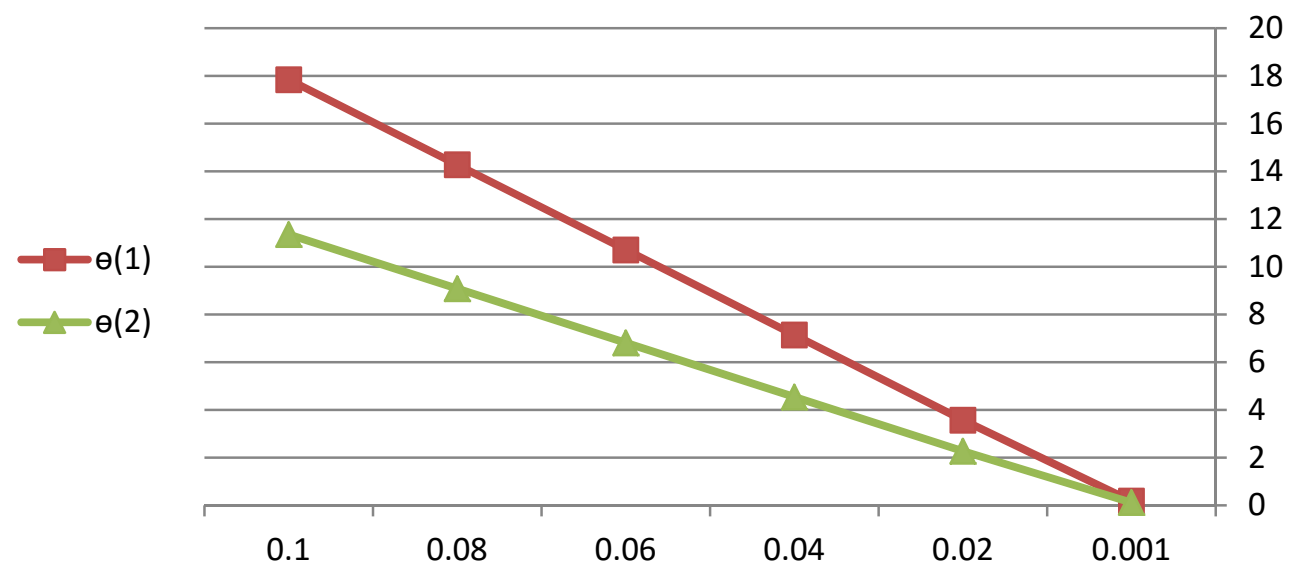

Figure 9. Vertical axis is angle and horizontal axis is electric current in fixed field of 3 Tesla and diagram of first and second frames angle changes is different in electric currents.

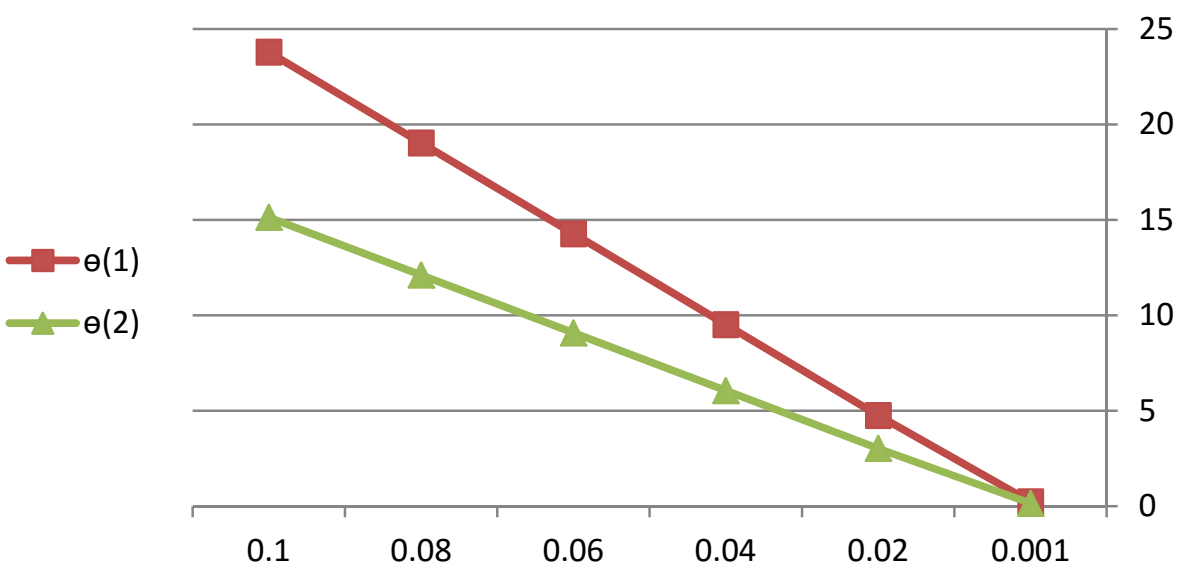

Figure 10. Vertical axis is angle and horizontal axis is electric current in fixed field of 4 Tesla and diagram of first and second frames angle changes is different in electric currents.

\section{$\theta$}

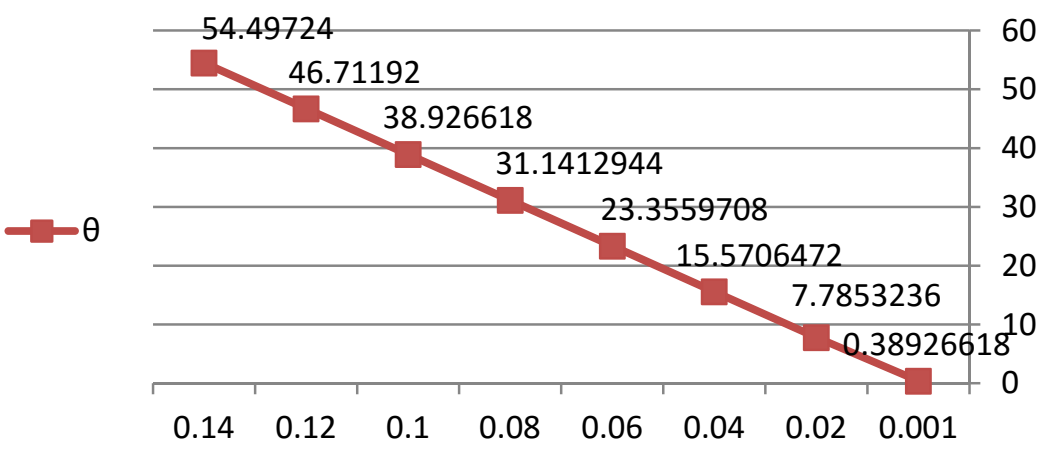

Figure 11. Amount of mirror surface changes in the field of 4 Tesla and between the current of 0.001-0.14 Amper Resonance frequency, is the frequency in which there is the maximum rotation in frame and diagram in this 
frequency in first and second frame is like below which it will happen for first frame in frequency of $30 \mathrm{~Hz}$ and for second frame in $25 \mathrm{~Hz}$ and because the coil of first and second frame will separate so in this plan we can apply for two different frequencies.

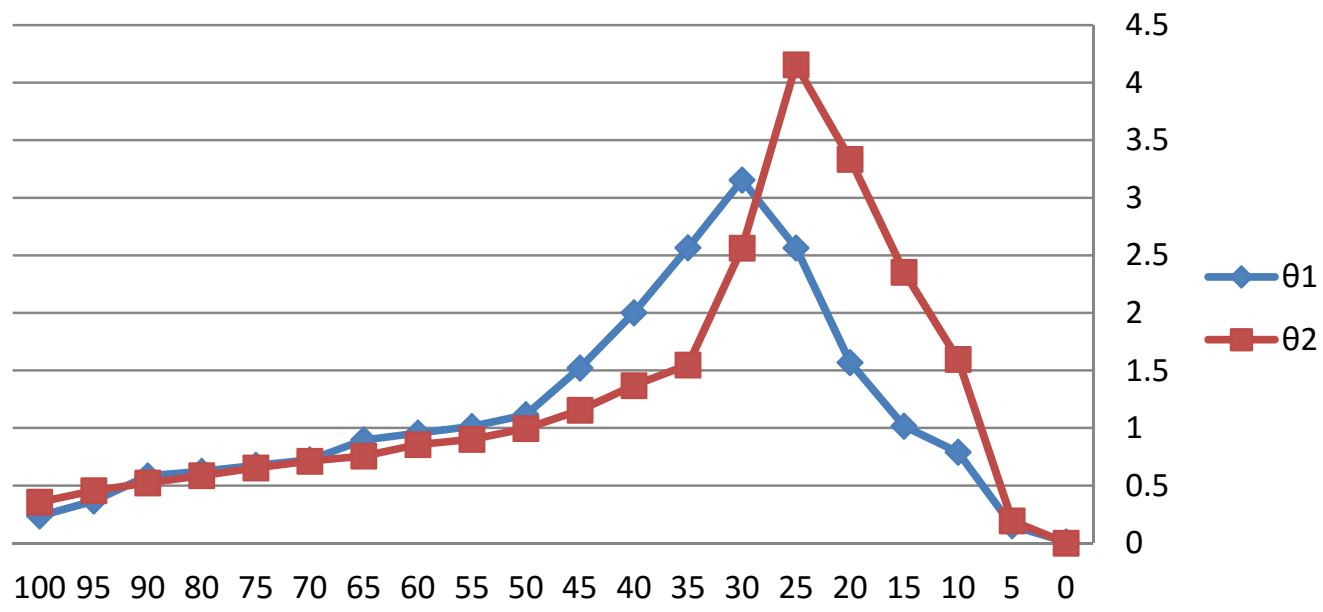

Figure 12. Frequency response to angle changes amount in two internal and external frames

\section{Suggestions}

Having micro dimensions is one of the features of this project and it has high medical and industrial applications also it will provide the rotation of micro mirror in micro dimensions which will capable of designing scan in small dimensions with high precision and other feature of this plan is the study of magnetic field in the fields that are used in CT scans and MRI instruments and human body and organisms can tolerate in this magnetic field and on the other hand this is the low resonance frequency of this plan and the maximum rotation of the mirror is in the \pm 54.49724 angle. Due to importance of scanner and amount of mirror's rotation and also in case of economic view we can use other conductors like aluminum, copper or other conductors than gold.

\section{References}

David, K. C. (1989). Field and wave electromagnetics, Edition: 2nd, January 11, 1989.

Fereudoni, A., \& Vahidi, B. (2000). Analysis of effects of very low frequency magnetic field in high voltage power lines on the human body in open area and in high voltage power, fourth conference of power plants, Amirkabir Industrial University of Tehran, Iran. MRI for technologist,woodward, peggy, Edition: $2^{\text {nd, }}$ November 2, 2000.

FUJITA, T., YOSHIDA, N., HASHINO, Y., MAENAKA, K., \& TAKAYAMA, Y. (1992). OPTICAL FEEDBACK SYSTEM FOR MEMS MIRROR CONTROL, 2167 Shosha, Himeji, Hyogo 671-2280, Japan.

Isheil-Bubaker, H., Fontaine, J. F., Rahel, R., Roy, M., \& Serri, J. (2013). 3D displacements and strains solid measurement based on the surface texture with a scanner laser, 8th CIRP Conference on Intelligent Computation in Manufacturing Engineering.

Kah, H. K., Takesh, K., Hsiao, F. L., \& Chengkuo, L. (2009). A 2-D MEMS Scanning Mirror Using Piezoelectric PZT Beam Actuators ,Proceedings of the Eurosensors XXIII conference.

Kah, H. K., Takeshi, K., \& Huicong, L. (2011). Investigation of a Piezoelectric Driven MEMS Mirror based on Single S-shaped PZT Actuator, Eurosensors XXV, September 4-7, 2011, Athens, Greece.

Kuijpers, A. A., Lierop, D., \& Sanders, R. H. M. (2009). Towards embedded control for resonant scanning MEMS micromirror, Proceedings of the Eurosensors XXIII conference.

Li, J., Chen, F., Shen, Q., Jiang, H., \& Zhang, L. (2007). Fabrication and dielectric properties of Si3N4-MgOAl2O3 by spark plasma sintering technique, Materials Science-Poland, 25(3).

Magnetic Properties of Materials (2011). Richard A. Clarke, Ee.surrey.ac.uk. Retrieved November 8, 2011, from www.microchem.com

Namazu, T. S., Inoue, K., Takio, T., Fujita, K. M., \& Koterazawa, K. (2005). VISCO-ELASTIC PROPERTIES OF MICRON-THICK SU-8 POLYMERS MEASURED BY TWO DIFFERENT TYPES OF UNIAXIAL 
TENSILE TESTS,Micro Electro Mechanical Systems. MEMS 2005. 18th IEEE International Conference.

Robert, R., David, H., Kenneth, \& Krane, S. (1992). Physics, 4th Edition, 1

Takayuki, F., Kazusuke, M., \& Yoichiro, T. (2005). Dual-axis MEMS mirror for large deflection-angle using SU-8 soft torsion beam, sciencedirect.

Takayuki, Fujita, Y., \& Nagatani, K. M. (2010). MEMS Mirror Controlling System with Holed-PSD.ICETET '10 Proceedings of the 2010 3rd International Conference on Emerging Trends in Engineering and Technology, 446-449

Takayuki, I., Mikio, O., \& Takashi, S. (2010). Shrinking design of a MEMS optical scanner having four torsion beams and arms, Sensors and Actuators A: Physical, 164(1), 95-106.

The Reliability of the Silicon Nitride Dielectric in Capacitive Mems Switches, Abuzer Dogan, Submitted in Partial Fulfillment of the Requirements for the Degree of Master of Science, August 2005.

Winter, C., Fabre, L., \& Conte, L. F. (2009). Micro-Beamer based on MEMS micro-mirrors and laser light source,Proceedings of the Eurosensors XXIII conference.

\section{Copyrights}

Copyright for this article is retained by the author(s), with first publication rights granted to the journal.

This is an open-access article distributed under the terms and conditions of the Creative Commons Attribution license (http://creativecommons.org/licenses/by/3.0/). 